\title{
Neutrino Signals from Galactic Binaries
}

\author{
Hugo R. Christiansen \\ Nucleo de Física, Universidade Estadual do Ceará, Av. Paranjana 1700, 60740-000 Fortaleza - CE, Brazil
}

\author{
Received on 19 October, 2006
}

\begin{abstract}
We analyze the possible origin of high-energy neutrinos coming from galactic sources. A model is proposed for neutrino hadroproduction in binary systems. Our results show that neutrino observatories like IceCube can establish whether $\mathrm{TeV}$ neutrinos and gamma rays emitted by microquasars are the decay products of pions. Such pions can be produced where the jet collides with the stellar wind of the companion star giving rise to hadronic interactions. We improve previous predictions by considering energy dependent pion multiplicities, particularly significant at high energies, and the most recent values for the parameters of the system. We also discuss the gamma-ray flux associated with neutrino emission.
\end{abstract}

Keywords: Astro neutrinos; Galactic sources

\section{INTRODUCTION}

The origin of cosmic rays is a persistent problem of Astrophysics which has not yet been solved. It is therefore an important challenge to investigate the possible mechanisms behind astroparticle production. Among these, neutrinos are especially interesting for they unequivocally indicate the direction of the source. Astrophysical sources of high-energy neutrinos are expected to contain different radiation and matter fields among which relativistic hadrons. Neutron stars (NS) are well-studied particle accelerators that can bring hadrons up to very high energies. When a NS belongs to a binary system, the accelerated hadrons can collide with convenient targets in the companion star. X-ray binaries are thus potential high-energy neutrino sources (see [1] and references therein). The magnetosphere of X-ray binaries presents electrostatic gaps that can disrupt the accretion process around the NS and strongly accelerate protons. These protons, moving along closed field-lines, can impact on the accreting material producing gamma-rays [2, 3] and neutrinos [4, 5].

When the magnetic field of the neutron star is not too intense, ejection phenomena can take place. The X-ray binary is then called 'microquasar' and a bipolar jet is displayed. A small fraction of the jet components consist of relativistic hadrons (e.g. Sco X-1 [6] and LS I +61 303 [7, 8]). Microquasars can be powered either by a weakly magnetized neutron star or by a black hole (e.g. Cygnus X-1). Some of them are suspected to be gamma-ray sources [9-12] and can presumably lead also to neutrino production through photohadron $[13,14]$ or proton-proton [15-18] interactions.

Microquasars with a Be type primary present slow and dense equatorial winds that form a circumstellar disk around it. It is precisely therein that the compact object (NS or $\mathrm{BH}$ ) moves around and the interactions occur. We will study how the interaction of the jet with this circumstellar material can lead to a prominent neutrino source. We shall focus on a specific object, LS I +61303 , for which all basic parameters are rather well-determined in order to make quantitative predictions that can be tested with the new generation of neutrino telescopes (IceCube, in this case [19]). Our results, however, will have general interest for any microquasar with nonspherically symmetric winds. For a comprehensive discussion see Christiansen et al. (2006) [20].

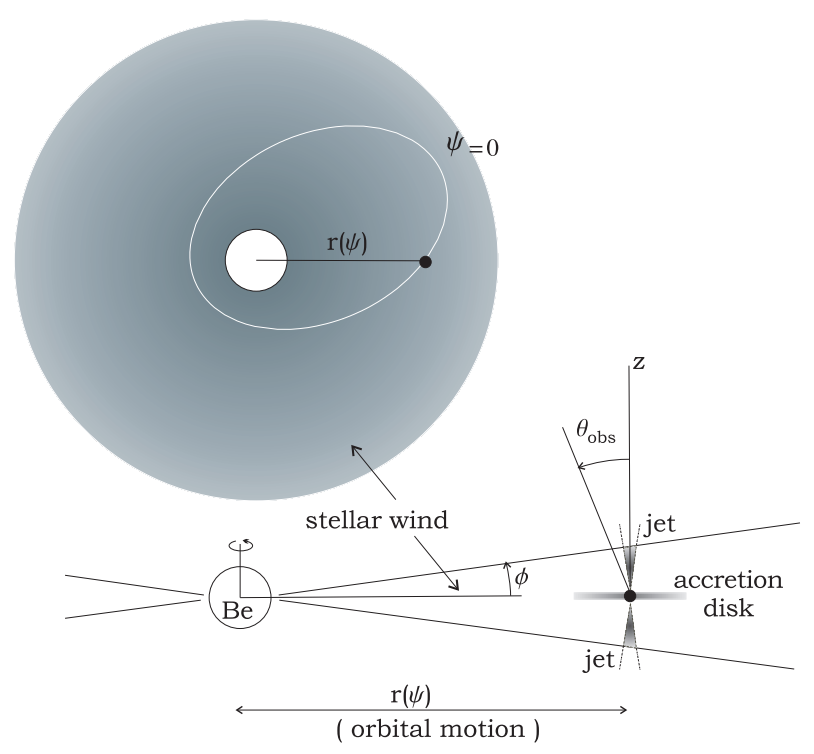

FIG. 1: A sketch of the binary system and its elements.

\section{THE SOURCE}

Binary systems, consisting of a massive star and a compact object (CO hereafter), are likely to emit high-energy neutrinos. We will consider a primary star of the B-type, generating a radially outflowing wind, with a secondary neutron star moving around in a Keplerian orbit. Their relative position is given by $r(\psi)=a\left(1-e^{2}\right) /(1-e \cos (\psi))$, where $\psi$ is the orbital phase, $a$ is the semi-major axis of the ellipse and $e$ its eccentricity.

In the case of microquasar LS I +61 303 [21], which we will focus in particular, the most recent determination of the orbital parameters [22] indicates that the eccentricity of the system is $e=0.72 \pm 0.15$ and that the orbital inclination is $\sim$ $30^{\circ} \pm 20^{\circ}$ (the orbital axis length results from the star masses and the orbital period). The best determination of the orbital 
period ( $P=26.4960 \pm 0.0028$ days $)$ comes from radio data [23] (note that here $\psi$ is not the radio phase, which amounts to $0.232 \pi$ at the periastron [22]). The primary star is a B0 V with a dense, nearly equatorial, wind that defines a circumstellar disk with a half-opening angle $\phi=15^{\circ}$. Its density is given by $\rho_{\mathrm{w}}(r)=\rho_{0}\left(r / R_{*}\right)^{-n}$, where $\rho_{0}=10^{-11} \mathrm{~g} \mathrm{~cm}^{-3}$, and the wind velocity thus follows as $v_{\mathrm{W}}=v_{0}\left(r / R_{*}\right)^{n-2}$. We will adopt $v_{0}=$ $5 \mathrm{~km} / \mathrm{s}, R_{*}=10 R_{\odot}, M_{*}=10 M_{\odot}$, and $n=3.2[24,25]$.

The wind accretion rate onto the $\mathrm{CO}$ is

$$
\dot{M}_{\mathrm{c}}=\frac{4 \pi\left(G M_{\mathrm{c}}\right)^{2} \rho_{w}(r)}{v_{\text {rel }}^{3}},
$$

where $M_{\mathrm{c}}=1.4 M_{\odot}$ is the $\mathrm{CO}$ mass and $v_{\text {rel }}$ is its velocity relative to the circumstellar wind.

Recently, Massi et al. (2001) [7] have detected the existence of relativistic radio jets in LS I +61303 extending to about $400 \mathrm{AU}$ from the compact object [26].

The matter content of microquasar jets is unknown, although the presence of ions in the jets of SS 433 has been proved by iron X-ray line observations [27-29]. We will therefore assume the existence of relativistic protons in the observed jets of LS I +61303 .

The jet kinetic-power $Q_{\mathrm{j}}$ is coupled to $\dot{M}_{\mathrm{c}}$ by $Q_{\mathrm{j}}=q_{\mathrm{j}} \dot{M}_{\mathrm{c}} c^{2}$ [30]. In the jet-disk symbiosis model, $q_{\mathrm{j}} \sim 0.1$ for microquasars in the low-hard state [31]. Most of the jet power consists of cold protons ejected with a macroscopic Lorentz factor $\Gamma \sim 1.25$ [7] which keep the relativistic hadrons in the jet [32]. We shall assume that for highly relativistic hadrons in the jet $q_{\mathrm{j}}=10^{-2}$.

The jet shall be conical with its axis, $z$, normal to the orbital plane. Its radius is $R_{\mathrm{j}}(z)=z R_{0} / z_{0}$, where $z_{0}=10^{7} \mathrm{~cm}$ is the injection point and $R_{0}=z_{0} / 10$ is the initial radius of the jet (see [15] and [11] for additional details). In Figure 1 we show a sketch of the general situation.

\section{THE INTERACTION}

The relativistic proton flux $J_{p}^{\prime}\left(E_{p}^{\prime}\right)$ has a power-law spectrum characterized by $\alpha$, whose $z$ dependence is given by $J_{p}^{\prime}\left(E_{p}^{\prime}\right)=\frac{c K_{0}}{4 \pi}\left(z_{0} / z\right)^{2} E_{p}^{\prime-\alpha}$ [33] (the prime refers to the jet frame). In the lab (observer) frame it can be written as [34]

$J_{p}\left(E_{p}, \theta\right)=\frac{c K_{0}}{4 \pi}\left(\frac{z_{0}}{z}\right)^{2} \frac{\Gamma^{-\alpha+1}\left(E_{p}-\beta_{\mathrm{b}} \sqrt{E_{p}^{2}-m_{p}^{2} c^{4}} \cos \theta\right)^{-\alpha}}{\sqrt{\sin ^{2} \theta+\Gamma^{2}\left(\cos \theta-\frac{\beta_{\mathrm{b}} E_{p}}{\sqrt{E_{p}^{2}-m_{p}^{2} c^{4}}}\right)^{2}}}$.

Here $\beta_{\mathrm{b}}$ is the bulk velocity in units of $c$ and $\theta$ is the angle subtended by the proton velocity direction and the jet axis. The normalization constant $K_{0}$ is related to the number density $n_{0}{ }^{\prime}$ of particles flowing in the jet at $R_{0}$ and can be determined as in [15].

As long as the particle gyro-radius is smaller than the radius of the jet, the matter from the wind can penetrate the jet diffusing into it. This process can generate high-energy photons and neutrinos from $p p$ collisions and subsequent decays. Penetration imposes a constraint onto the value of the magnetic field in the jet: $B_{\text {jet }} \geq E_{\mathrm{k}}^{\mathrm{p} \text {, wind }} /(e R)$, where $E_{k}^{\mathrm{p} \text {, wind }}=m_{p} v_{\mathrm{rel}}^{2} / 2$ is the kinetic energy of the cold protons in the slow stellar wind. For $E_{\mathrm{k}}^{\mathrm{p} \text {, wind }}$ maximum, at periastron, it results $B_{\text {jet }} \geq 2.810^{-6}$ $\mathrm{G})$ which, as we will see, is assured. Since some effects, like shock formation on the boundary layers, could prevent some particles from entering into the jet, one has to consider a penetration factor $f_{\mathrm{p}}$ that takes into account particle rejection. We will adopt $f_{\mathrm{p}} \sim 0.1$, in order to reproduce the observed gamma-ray flux at $\mathrm{GeV}$ energies, where opacity effects due to pair creation are unimportant. A value of $\alpha=2.2$ characterizes the power law spectrum matching the GeV gammaray spectrum observed by EGRET [35]. Note that $\theta$ will be roughly the same as that of the emerging photon $\left(\theta \approx \theta_{\text {obs }}\right)$ [22].

\section{HADRO-PRODUCTION OF NEUTRINOS}

High-energy pions, from which high-energy photons and neutrinos arise, can be produced in relativistic proton collisions in both $p p$ and $p \gamma$ channels. In any case, photon and neutrino production are closely related. The $v_{\mu}+\bar{v}_{\mu}$ differential neutrino flux $\left(d N_{\mathrm{v}} / d E_{\mathrm{v}}\right)$ can be simply obtained from the differential $\gamma$-ray flux $\left(d N_{\gamma} / d E_{\gamma}\right)$ by means of an identity derived from the conservation of energy [36-38]

$$
\int_{E_{\gamma}^{\min }}^{E_{\gamma}^{\max }} E_{\gamma} \frac{d N_{\gamma}}{d E_{\gamma}} d E_{\gamma}=D \int_{E_{\mathrm{v}}^{\min }}^{E_{\mathrm{v}}^{\max }} E_{\mathrm{v}} \frac{d N_{\mathrm{v}}}{d E_{\mathrm{v}}} d E_{\mathrm{v}} .
$$

The relationship between both integrals is given by $D$ and depends on the energy distribution among the particles resulting from the inelastic collision. As a consequence, the associated flux of neutrinos, which arise from charged pions, will depend on whether the $\pi^{0}$ 's are of $p p$ or $p \gamma$ origin. Its value can be obtained from routine particle physics and some kinematics assumptions. In $p \gamma$ processes, most of the energy is transferred to leptons, radiating plenty of photons but no high energy neutrinos. In $p p$ interactions, $1 / 3$ of the proton energy goes into each pion flavor, on average, and thus two muonneutrinos (and two muon-antineutrinos) are produced in the charged channel with energy $E_{\pi} / 4$, for every photon with energy $E_{\pi} / 2$ in the neutral channel. Therefore the energy in neutrinos matches the energy in photons and $D=1$.

The relevant parameters to relate neutrino and $\gamma$-ray fluxes are the maximum and minimum energies of the produced photons and neutrinos, appearing as integration limits in Eq. (3). The maximum neutrino energy results from the maximum energy of the accelerated protons $\left(E_{p}^{\max }\right)$ which is related to the maximum $\gamma$-ray energy observed. Thus, for the $p p$ case one conservatively obtains

$$
E_{p}^{\max }=6 E_{\gamma}^{\max }, \quad E_{\mathrm{v}}^{\max }=\frac{1}{2} E_{\gamma}^{\max } .
$$

The minimum gamma and neutrino energies are fixed by the threshold for pion production. For the $p p$ case $E_{p}^{\min }=$ 
$\Gamma\left[\left(2 m_{p}+m_{\pi}\right)^{2}-2 m_{p}^{2}\right] / 2 m_{p}$, where $\Gamma$ is the Lorentz factor of the accelerator relative to the observer. The average minimum neutrino energy is obtained from $E_{p}^{\mathrm{min}}$ using the same relations of Eq. (4). When purely hadronic collisions are considered, pion production becomes the natural production channel. Relativistic protons in the jet will interact with target protons in the wind through the reaction channel $p+p \rightarrow p+p+\xi_{\pi^{0}} \pi^{0}+\xi_{\pi^{ \pm}}\left(\pi^{+}+\pi^{-}\right)$. Then, pion decay chains lead to gamma-ray and neutrino emission. Isospin symmetry, which is in agreement with Fermis original theory of pion production relates the three multiplicities with an equal value that we simply refer as $\xi_{\pi}$.

\section{MULTIPLICITY}

When we consider energy dependent multiplicities, the relation between energy maxima is quite different from Eq.(4). According to Ginzburg \& Syrovatskii (1964) [39], for inelastic $p p$ interactions we can obtain the gamma-ray energy from the proton energy by

$$
E_{p} \approx 6 \xi_{\pi}\left(E_{p}\right) E_{\gamma} / K .
$$

The inelasticity coefficient is $K \sim 0.5$ since on average a leading nucleon and a pion cloud leave the interaction fireball each carrying half of the total incident energy (see ref. [40] for a discussion on this coefficient). For the energy dependent pion multiplicity we will follow the prescription adopted by Mannheim and Schlickeiser (1994) [41]

$$
\xi_{\pi} \simeq\left(E_{p} / \mathrm{GeV}-1.22\right)^{1 / 4},
$$

which should be valid up to $10^{4} \mathrm{GeV}$. Since for higher energies we have no data, we shall adopt a softer root growing law for $\xi_{\pi}\left(E_{p}\right)$ which leads to a more reliable relationship between neutrino and proton energies. According to [42], based on Ref.[43], the multiplicity should be kept below 15 for $E_{p}=100 \mathrm{TeV}$. In order to analytically lessen Eq. (6) to such values, we can interpolate the 10 to $100 \mathrm{TeV}$ range in a simple way with a $1 / 5$ fractional power. This gives $\xi_{\pi} \approx 14$ for $E_{p}=100 \mathrm{TeV}$. For the next two decades, there are no confident approaches so we extrapolate $\xi_{\pi}$ with a $1 / 6$ root up to the $\mathrm{PeV}$ maximal proton energies obtained in Eq.(9). This gives $\xi_{\pi} \approx$ 26 for $E_{p}=50 \mathrm{PeV}$ (periastron). Notice that a continuous expression for $\xi_{\pi}$ is needed in order to evaluate both luminosity and neutrino signal from Eq.(12) and Eq.(16). Notwithstanding, the corresponding integrals are not really sensitive to this analytical choice since the multiplicity effects are actually weak: on the one hand, $\sigma_{p p}$ depends only logarithmically on $\xi_{\pi}$; on the other, it modifies the upper limit of the integrals, but then again as the integrand is quite steep the contribution of the queue in a larger domain is below a few percent. Note finally that here we conservatively adopted a log instead of a $\log ^{2} E_{p}$ - dependence of the cross section. For the highest proton energies, as given by Eq.(9), one gets $E_{v}^{\max } \sim \frac{1}{156} E_{p}^{\max }$ at periastron (see Fig.2b). We will promptly see that one can in fact expect for a significant signal to noise relationship in less than one year of operational time of a ' $\mathrm{km}^{3}$ ' telescope.

\section{PROTON ACCELERATION}

As mentioned in [1], the maximum energy at which a particle can be accelerated, can be derived from the simple argument that the Larmor radius of the particle should be smaller than the size of the acceleration region [44]. Since energy losses inside LSI can be neglected, this maximum energy $E_{15}^{M}$ (in units of $10^{15} \mathrm{eV}$ ) is related to the strength of the magnetic field $B$ (in units of Gauss) and the size of the accelerating region $R$ (in units of $\mathrm{cm}$ ) by the following relationship

$$
E_{15}^{M} \sim 0.310^{-12} \beta Z B_{G} R_{\mathrm{cm}}
$$

where $\beta$ is the velocity of the shock wave or the acceleration mechanism efficiency, and $Z$ is the charge. Diffusive particle acceleration through shocks may occur in many candidate sites. In the case of LS I +61 303, we adopt quasi-parallel shocks and $B$ given by equipartition with the cold, confining plasma [32]).

Assuming that both matter and field follow adiabatic evolution when moving along the jet, we can assume equipartition between the magnetic field energy and the kinetic energy of the jet. Then, the magnetic field in the jet reference frame reads [45][32]

$$
B(z, \psi)=\frac{4 G M_{\mathrm{c}}}{R} \sqrt{\pi q_{\mathrm{j}} \rho_{w} v_{\mathrm{j}} / v_{\mathrm{rel}}^{3}},
$$

where $v_{\mathrm{j}}=c \beta_{\mathrm{b}}$ is the jet cold proton velocity so that here $q_{\mathrm{j}} \sim$ 0.1 . Now, the maximum proton energy compatible with this magnetic field can be obtained by means of the gyro-radius identity

$$
E_{p}^{\max }(\psi)=\mathrm{e} R B
$$

This value for $E_{p}^{\max }$ will be used in the next section to calculate gamma-ray and neutrino intensities.

In Figure 2 we show the magnetic field (a) and maximum proton energy (b) in the jet in terms of the orbital position for LS I +61 303. As we can see in the figure, the maximum proton energy produced amounts to more than $50 \mathrm{PeV}$ at periastron and lies below $15 \mathrm{PeV}$ near apastron. Note that the minimum does not occur exactly at apastron due to the particular orbital variation of the accretion rate. Furthermore, there is a second sharp maximum, readily identified with the function $1 / v_{\text {rel }}^{3}$, which grows dramatically when the compact object gets far from the primary star and moves more parallel to the weak stellar wind.

\section{RELATED GAMMA RADIATION}

Regarding gamma-rays production, since the branching ratio of charged pions into photons (plus leptons) is about 6 orders of magnitude smaller than that of $\pi^{0}$ 's into photons (alone) [46] we should concentrate on the neutral parents.

We will make all calculations in the lab frame, where the cross sections for proton interactions have suitable parameterizations. Neutral pion decay after high-energy proton 

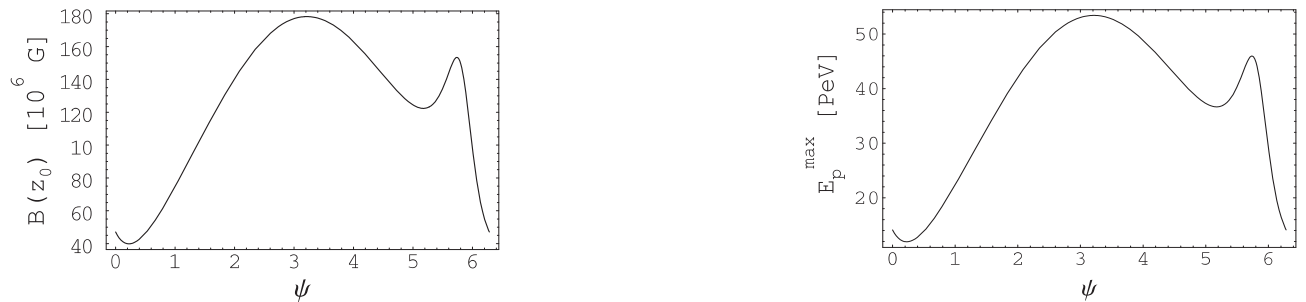

FIG. 2: Left (a): Maximal magnetic field in the jet, $B^{\max }(\psi)$, as a function of the orbital position of the compact object (in Gauss). Right (b): Highest proton energy compatible with the magnetic field (in PeV) as a function of $\psi$ (see Ref.[20]).

collisions is a natural channel for high-energy neutrino and gamma-ray production (see also Christiansen et al. 2006b [47]).

The differential gamma-ray emissivity from $\pi^{0}$-decays can be expressed as (e.g. Aharonian \& Atoyan 1996 [48]):

$$
q_{\gamma}\left(E_{\gamma}, \theta\right)=4 \pi \eta_{\mathrm{A}} \sigma_{p p}\left(E_{p}\right) \frac{2 Z_{p \rightarrow \pi^{0}}^{(\alpha)}}{\alpha} J_{p}\left(E_{\gamma}, \theta\right),
$$

(in $\mathrm{ph} \mathrm{s}^{-1} \mathrm{sr}^{-1} \mathrm{erg}^{-1}$ ), where $Z_{p \rightarrow \pi^{0}}^{(\alpha)}=0.092$ is the so-called spectrum-weighted moment of the inclusive cross-section and it is related to the fraction of kinetic proton energy transferred to the pions (see Gaisser 1990 [40]). The parameter $\eta_{\mathrm{A}}$ takes into account the contribution from different nuclei in the wind. For standard composition of cosmic rays and interstellar medium $\eta_{\mathrm{A}} \sim 1.4$. The proton flux distribution $J_{p}\left(E_{\gamma}\right)$ (see eq.(2)) is evaluated at $E=E_{\gamma}$, and $\sigma_{p p}\left(E_{p}\right) \approx$ $30\left[0.95+0.06 \log \left(E_{p} / \mathrm{GeV}\right)\right](\mathrm{mb})[48]$ is the cross section for inelastic $p p$ interactions at energy $E_{p} \approx 6 \xi_{\pi^{0}} E_{\gamma} / K$, for $E_{\gamma} \geq 1 \mathrm{GeV}$.

The spectral gamma ray intensity (in $\mathrm{ph} \mathrm{s}^{-1} \mathrm{erg}^{-1}$ ) is

$$
I_{\gamma}\left(E_{\gamma}, \theta\right)=\int_{V} d^{3} \vec{r} n(\vec{r}) q_{\gamma}\left(E_{\gamma}, \theta\right)
$$

where $V$ is the interaction volume between the jet and the circumstellar disk. The particle density of the wind that penetrates the jet is $n(r) \approx f_{\mathrm{p}} \rho_{w}(r) / m_{p}$, and the generated luminosity in a given energy band results

$$
L_{\gamma}\left(E_{\gamma}^{a, b}, \theta\right)=\int_{E_{\gamma}^{a}}^{E_{\gamma}^{b}} d E_{\gamma} E_{\gamma} I_{\gamma}\left(E_{\gamma}, \theta\right)
$$

The issue of gamma-ray emission is further analyzed in Romero et al. 2005 [49].

\section{NEUTRINO FLUX AND SIGNAL}

In order to obtain the spectral flux of neutrinos, we need an explicit expression for the neutrino emissivity with all the details therein. Instead, we can take eq. (3) so as to extract an expression for $I_{v}\left(E_{v}\right)$. Now, we write eqs. (3) and (4) as

$$
L_{v}=D L_{\gamma}, 2 E_{\mathrm{v}}=E_{\gamma},
$$

which result from the assumption that, together, one $(\mu)$ neutrino and one anti-neutrino carry half one charged-pion energy (note that the number of $(\mu)$ neutrinos resulting from $\pi^{+}$and $\pi^{-}$decays is equal to the number of gamma rays coming from $\pi^{0}$ since the number of pions produced is the same for the three flavors; same holds for the flux of $(\mu)$ anti-neutrinos).

From Eq.(13) we obtain

$$
I_{v}\left(E_{\mathrm{v}}\right)=4 I_{\gamma}\left(E_{\gamma}=2 E_{\mathrm{v}}\right),
$$

assuming $p p$ interactions where $1 / 3$ of the proton energy goes to each pion flavor. With this, the neutrino intensity reads

$$
I_{V}\left(E_{V}, \theta\right)=4 \int_{V} d V \frac{f_{p} \rho_{w}(r)}{m_{p}} q_{\gamma}\left(\vec{r}, 2 E_{V}, \theta\right)
$$

Now, we compute the convolution of the neutrino flux with the event probability. For signal and noise above $1 \mathrm{TeV}$ we obtain respectively

$S=T_{\mathrm{obs}} A_{\mathrm{eff}} \frac{1}{4 \pi d^{2}} \frac{1}{2 \pi} \int_{0}^{2 \pi} d \psi \int_{1 \mathrm{TeV}}^{E_{\mathrm{v}}^{\max }(\psi)} d E_{\mathrm{v}} I_{v}\left(E_{\mathrm{v}}, \psi\right) P_{\mathrm{v} \rightarrow \mu}\left(E_{\mathrm{v}}\right)$,

and

$N=\left[T_{\mathrm{obs}} A_{\mathrm{eff}} \Delta \Omega \frac{1}{2 \pi} \int_{0}^{2 \pi} d \psi \int_{1 \mathrm{TeV}}^{E_{\mathrm{v}}^{\max }(\psi)} d E_{\mathrm{v}} F_{B}\left(E_{\mathrm{v}}\right) P_{\mathrm{v} \rightarrow \mu}\left(E_{\mathrm{v}}\right)\right]^{1 / 2}$,

where $T_{\mathrm{obs}}$ is the observational time period, $A_{\mathrm{eff}}$ is the effective area of the detector, $d$ is the distance to the system, and $\Delta \Omega$ is the solid angle of the search bin. The function

$$
F_{B}\left(E_{\mathrm{v}}\right) \leq 0.2\left(E_{\mathrm{v}} / \mathrm{GeV}\right)^{-3.21} \mathrm{GeV}^{-1} \mathrm{~cm}^{-2} \mathrm{~s}^{-1} \mathrm{sr}^{-1}
$$

represents the $v_{\mu}+\bar{v}_{\mu}$ atmospheric flux (see Volkova 1980, and Lipari 1993 [50, 51]), and

$$
P_{\mathrm{v} \rightarrow \mu}\left(E_{\mathrm{v}}\right)=1.310^{-6}\left(E_{\mathrm{v}} / \mathrm{TeV}\right)^{0.8}
$$

is the probability that a neutrino of energy $E_{\mathrm{V}} \sim 1-10^{3} \mathrm{TeV}$, on a trajectory through the detector produces a muon (see Gaisser et al. 1995 [52]).

As we have already seen in Sect.V, the value of the maximal neutrino energy is a function of orbital position of the jet and is given by

$$
E_{v}^{\max }(\psi) \approx \frac{K}{12 \xi_{\pi}(\psi)} E_{p}^{\max }(\psi)
$$

Our estimate for this expression is $E_{\mathrm{v}}^{\max } \approx 85 \mathrm{TeV}$ at periastron, and about $20 \mathrm{TeV}$ at apastron (see Fig.3). 


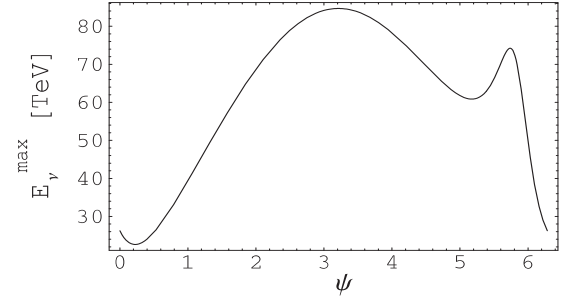

FIG. 3: Maximal neutrino energies $E_{\mathrm{v}}^{\max }$ along the orbit (in TeV).

If we now set the features of a km-scale detector such as IceCube [19] in the model $\left(A_{\mathrm{eff}}=1 \mathrm{~km}^{2}\right.$ and $\left.\Delta \Omega \approx 310^{-4} \mathrm{sr}\right)$, and the distance to LS I $+61303(d=2 \mathrm{kpc})$ we get

$$
S /\left.N\right|_{1 \text { year }} \geq 9.42
$$

for the signal to noise ratio in a one-year period of observation. This is consistent with the upper limit from AMANDA-II experiment [53].

Note that the present prediction is restricted to $\mu$-neutrino production at the source. As a matter of fact, there is nowadays strong experimental evidence of the existence of neutrino oscillations which occur if neutrinos are massive and mixed. The expected ratios at sources of high-energy neutrino fluxes from $p p$ collisions are $1: 2: 10^{-5}$ for the $e, \mu, \tau$ neutrino flavors, in the range $1 \mathrm{GeV} \leq E_{\mathrm{v}} \leq 10^{12} \mathrm{GeV}$. The estimate is that these ratios become in average $1: 1: 1$ for $L(\mathrm{pc}) / \mathrm{E}(\mathrm{GeV}) \geq 10^{-10}$, where the distance is in parsecs (see Athar et al. 2005 [54]). Also recently, a detailed analysis of supernova remnants reported by Costantini and Vissani (2005) [55] claims a $50 \%$ of muon-neutrino plus muon anti-neutrino flux reduction due to flavor oscillations along astrophysical distances. Note finally, that the neutrino signal measured at Earth might be further attenuated due to matter absorption.

\section{LUMINOSITY AND OPACITY}

As a result of the discussion above, our predictions for both neutrino and gamma ray luminosities between $E_{v, \gamma}^{\min }=1,2$ $\mathrm{TeV}$ and $E_{v, \gamma}^{\max }$ amount to $510^{34} \mathrm{erg} \mathrm{s}^{-1}$ for a source such as LS I +61 303 .

The total neutrino luminosity is related to the spectral neutrino intensity $I_{v}\left(E_{v}\right)$ by means of

$$
L_{v}=\int_{E_{\mathrm{v}}^{\min }}^{E_{\mathrm{v}}^{\max }} d E_{\mathrm{v}} E_{\mathrm{v}} I_{v}\left(E_{\mathrm{v}}\right)
$$

as we mentioned in Sect. II. Since we do not have an expression for $I_{V}\left(E_{v}\right)$, we can use eq. (3) and relate it to the gamma ray luminosity $L_{\gamma}=\int_{E_{\gamma}^{\min }}^{E_{\max }} d E_{\gamma} E_{\gamma} I_{\gamma}\left(E_{\gamma}\right)$ which we know in more detail. Now, there is in fact an experimental constraint on this quantity, as recently analyzed by Fegan et al. (2005) [56]. There, it is claimed that, above $0.35 \mathrm{TeV}$, the total flux of gamma rays that can be produced must satisfy

$$
\frac{1}{4 \pi d^{2}} \int_{0.35 \mathrm{TeV}}^{\infty} d E_{\gamma} I_{\gamma}\left(E_{\gamma}\right) \leq 1.710^{-11} \mathrm{ph} \mathrm{cm}^{-2} \mathrm{~s}^{-1} .
$$

Our calculations for gamma rays give about four times this value (if we extrapolate our model to ultra high energies), so we need to show that most of the high-energy gamma rays get absorbed. Of course, the absorption mechanism should not modify the flux of neutrinos. Infrared photon fields can be responsible for $\mathrm{TeV}$ photon absorption in the source. To show it, we calculated the optical depth $\tau$ within the circumstellar disk. The optical depth remains above unity for a wide range of photon energies but a sharp knee takes place at $E_{\gamma} \approx 250 \mathrm{GeV}$. For TeV gamma energies it still has an important effect on the gamma-ray luminosity. In particular, the opacity-corrected total flux above $E_{\gamma}=350 \mathrm{GeV}$ drops from $7.1610^{-11}$ to 1.42 $10^{-12} \mathrm{ph} \mathrm{cm}^{-2} \mathrm{~s}^{-1}$, lying well below the Fegan et al.'s threshold. Other observing windows, however, could reveal the presence of a hadronic gamma-ray source at the position of LS I +61303. In particular, between 1 and $50 \mathrm{GeV}$ the opacity is sufficiently low as to allow a relatively easy detection by instruments like the ground-based MAGIC telescope and the LAT instrument of GLAST satellite. LS I +61303 is an outstanding candidate to corroborate that high-energy emission is a common property of microquasars. Its location, in addition, makes of it an ideal candidate for IceCube. A neutrino detection from this source would be a major achievement, which would finally solve the old question on whether relativistic protons are part of the matter content of the energetic outflows presented by accreting compact objects.

\section{CONCLUSIONS}

We have analyzed the possible origin of galactic neutrinos and related gamma radiation. We have particularly focused microquasar LS I +61 303 because it is a well-studied object which allows a reliable prediction of the neutrino flux. We showed that neutrino observatories like IceCube can establish whether $\mathrm{TeV}$ neutrinos and gamma rays emitted by microquasars are the decay products of pions. Such pions are produced in the hadronic jet when it collides with the wind generated by the massive star. Our predictions result from the most recent values of the parameters of the system and energy dependent pion multiplicities. Above $1 \mathrm{TeV}$, the mean-orbital $v$-luminosity obtained is about $510^{34} \mathrm{erg} / \mathrm{s}$. It can be related to an event rate of 4-5 $\mu$-type neutrinos per kilometer-squared per year even after maximal neutrino mixing is considered. The upper limit of integration depends on the orbital position and is a function of the largest magnetic gyro-radius compatible with the jet dimensions. The maximal neutrino energies will therefore vary between 20 and $85 \mathrm{TeV}$ along the orbit. Opacity effects on the associated gamma radiation result in a significant attenuation of the $\gamma$-ray signal at source compatible with experimental limits. Nevertheless, with instruments like MAGIC and GLAST, LS I +61303 is likely to be detectable at low $\mathrm{GeV}$ energies. This would be crucial to test current ideas of particle acceleration in compact objects.

\section{Acknowledgements}

H.R. Christiansen is grateful to Fundação de Amparo à Pesquisa do Ceará for full support of the expenses related to 
the meeting. H.R.C. is supported by CNPq and FUNCAP, Brazil.

[1] W. Bednarek, G. F. Burgio, and T. Montaruli, New. Astron. Rev. 49, 1 (2005).

[2] K. S. Cheng, et al., Astrophys. J. 390, 480 (1992).

[3] G. E. Romero, M. M. Kaufman Bernad, J. A. Combi, and D. F. Torres, Astron. and Astrophys. 376, 599 (2001).

[4] K.S. Cheng, T. Cheung, M.M. Lau, and K.W. Ng, J. Phys. G 18, 725 (1992).

[5] L.A. Anchordoqui, et al., Astrophys. J. 589, 481 (2003).

[6] R.P. Fender, et al., Nature 427, 222 (2004).

[7] M. Massi, M. Ribó, J.M. Paredes, et al., Astron. and Astrophys. 376, 217 (2001).

[8] M. Massi, Astron. and Astrophys. 422, 267 (2004).

[9] J. M. Paredes, J. Martí, M. Ribó, and M. Massi, Science 288 2340 (2000).

[10] M. M. Kaufman Bernadó, G. E. Romero, and F. Mirabel, Astron. and Astrophys. 385, L10 (2002).

[11] V. Bosch-Ramon, G.E. Romero, and J.M. Paredes, Astron. and Astrophys. 429, 267 (2005).

[12] F.A. Aharonian, et al., Science 309, 746 (2005).

[13] A. Levinson, and A. Waxman, Phys. Rev. Lett. 87, 171101 (2001).

[14] C. Distefano, D. Guetta, E. Waxman, and A. Levinson, Astrophys. J. 575, 378 (2002).

[15] G.E. Romero, D.F. Torres, M.M. Kaufman Bernadó, and F. Mirabel, Astron. and Astrophys. 410, L1 (2003).

[16] G.E. Romero, and M. Orellana, Astron. and Astrophys. 439 237 (2005).

[17] D.F. Torres, G.E. Romero, F. Mirabel, Chin. J. Astron. Astrophys. Suppl. 5, 183 (2005).

[18] W. Bednarek, Astrophys. J. 631, 466 (2005).

[19] IceCube 2002, http://icecube.wisc.edu/

[20] H.R. Christiansen, M. Orellana, and G.E., Romero, Phys. Rev. D73 063012 (2006); H. R. Christiansen, Phys. Scripta 127, 82 (2006).

[21] LS I +61303 has long been associated with a gamma-ray source. First with the COS-B source CG135+01, and later on with 3EG J0241+6103 (Gregory and Taylor 1978, Kniffen et al. 1998). The gamma-ray emission is clearly variable (Tavani et al. 1998) and has been recently shown that the peak of the gamma-ray lightcurve is consistent with the periastron passage (Massi 2004), contrary to what happens with the radio/X-ray emission, which peaks after the passage.

[22] J. Casares, I. Ribas, J.M. Paredes, et al., MNRAS 360, 1105 (2005).

[23] P.C. Gregory, Astrophys. J. 575, 427 (2002).

[24] J. Marti, and J.M. Paredes, Astron. and Astrophys. 298, 151 (1995).

[25] P.C. Gregory, and C. Neish, Astrophys. J. 580, 1133 (2002).

[26] M. Massi, M. Ribó, J.M. Paredes, et al. , Astron. and Astrophys. 414, L1 (2004)

[27] T. Kotani, N. Kawai, T. Aoki, et al., PASJ 46, L147 (1994).
[28] T. Kotani, N. Kawai, M. Matsuoka, and W. Brinkmann, PASJ 48, 619 (1996).

[29] S. Migliari, R. Fender, and M. Méndez, Science 297, 1673 (2002).

[30] H. Falcke, and P. L. Biermann, Astron. and Astrophys. 293, 665 (1995).

[31] R.P. Fender, MNRAS 322, 31 (2001).

[32] V. Bosch-Ramon, G.E. Romero, and J.M. Paredes, Astron. and Astrophys. 447, 263 (2006).

[33] G. Ghisellini, L. Maraschi, and A. Treves, Astron. and Astrophys. 146, 204 (1985).

[34] D. Purmohammad and J. Samimi, Astron. and Astrophys. 371, 61 (2001).

[35] R.C. Hartman, D.L. Bertsch, S.D. Bloom, et al., Astrophys. J. S. S. 123, 79 (1999).

[36] J. Alvarez-Muñiz, and F. Halzen, Astrophys. J. 576, L33-L36 (2002).

[37] F. W. Stecker, Astrophys. J. 228, 919 (1979).

[38] F. W. Stecker, and M. H. Salamon, Space Sci. Rev. 75, 341 (1996).

[39] V.L. Ginzburg, and S.I. Syrovatskii, Sov. Astron. 8, 342 (1964).

[40] T.K. Gaisser, Cosmic Rays and Particle Physics, Cambridge University Press, Cambridge 1990.

[41] K. Mannheim, and R. Schilckeiser, Astron. and Astrophys. 286, 983 (1994).

[42] M.C. Begelman, R. Bronislaw, M. Sikora Astrophys. J. 362, 38 (1990).

[43] C.D. Orth, and A. Buffington, Astrophys. J. 206, 312, (1976).

[44] A. M. Hillas, ARAA 22, 425 (1984).

[45] R.D. Blandford, and A. K onigl, Astrophys. J. 232, 34 (1979).

[46] S. Eidelman et al., Phys. Lett. B592, 1 (2004) and 2005 partial update for the 2006 edition. Review of Particle Physics, PDG, http://pdg.lbl.gov/2005/listings/mxxxcomb.html

[47] H.R. Christiansen, G.E. Romero and M. Orellana; Braz. J. Phys. bf 37 (2007), Proceedings of I LAWEP, ed. Gay Ducatti B., Porto Alegre 2005.

[48] F. A. Aharonian, and A. M. Atoyan, Astron. and Astrophys. 309, 917 (1996).

[49] G.E. Romero, H.R. Christiansen, M. Orellana, Astrophys. J. 632, 1093 (2005).

[50] L. V. Volkova, Yad. Fiz. 31, 1510, (1980) [1980, Sov. J. Nucl. Phys. 31, 784]

[51] P. Lipari, Astrop. Phys. 1, 195 (1993).

[52] T. K. Gaisser, F. Halzen, and T. Stanev, Phys. Rep. 258, 173 (1995) and references therein, Erratum-ibid. 271, 355 (1995).

[53] M. Ackermann, et al. Phys. Rev. D71, 077102 (2005).

[54] H. Athar, C.S. Kim, and J. Lee, Mod. Phys. Lett. 21, 1049 (2006).

[55] M.L. Costantini, and F. Vissani, Astrop. Phys. 23, 477 (2005).

[56] S. Fegan, et al., Astrophys. J. 624, 638 (2005). 